\title{
Poly(3,4-ethylenedioxythiophene) Bearing Pyridylboronic Acid Group for Specific Recognition of Sialic Acid
}

Wenfeng Hai, ${ }^{\dagger}$ Sihua Pu, ${ }^{\dagger}$ Xia Wang, ${ }^{\dagger}$ Liming Bao, $"$ Narenchaoketu Han, " Limei

Duan, ${ }^{\dagger}$ Jinghai Liu, ${ }^{*}, \dagger$ Tatsuro Goda, ${ }^{*}, \dagger, \S$ Wenming $\mathrm{Wu}^{*}, \dot{\dagger}$

$\dagger$ Inner Mongolia Key Laboratory of Carbon Nanomaterials, Nano Innovation Institute

(NII), College of Chemistry and Materials science, "College of Mongolian Medicine,

Inner Mongolia University for Nationalities, Tongliao 028000, China

ॠState Key Laboratory of Applied Optics, Chuangchun Institute of Optics, Fine

Mechanics and Physics, Chinese Academy of Sciences, Changchun 130033, China

${ }^{\S}$ Institute of Biomaterials and Bioengineering, Tokyo Medical and Dental University

(TMDU), 2-3-10 Kanda-Surugadai, Chiyoda, Tokyo 101-0062, Japan 


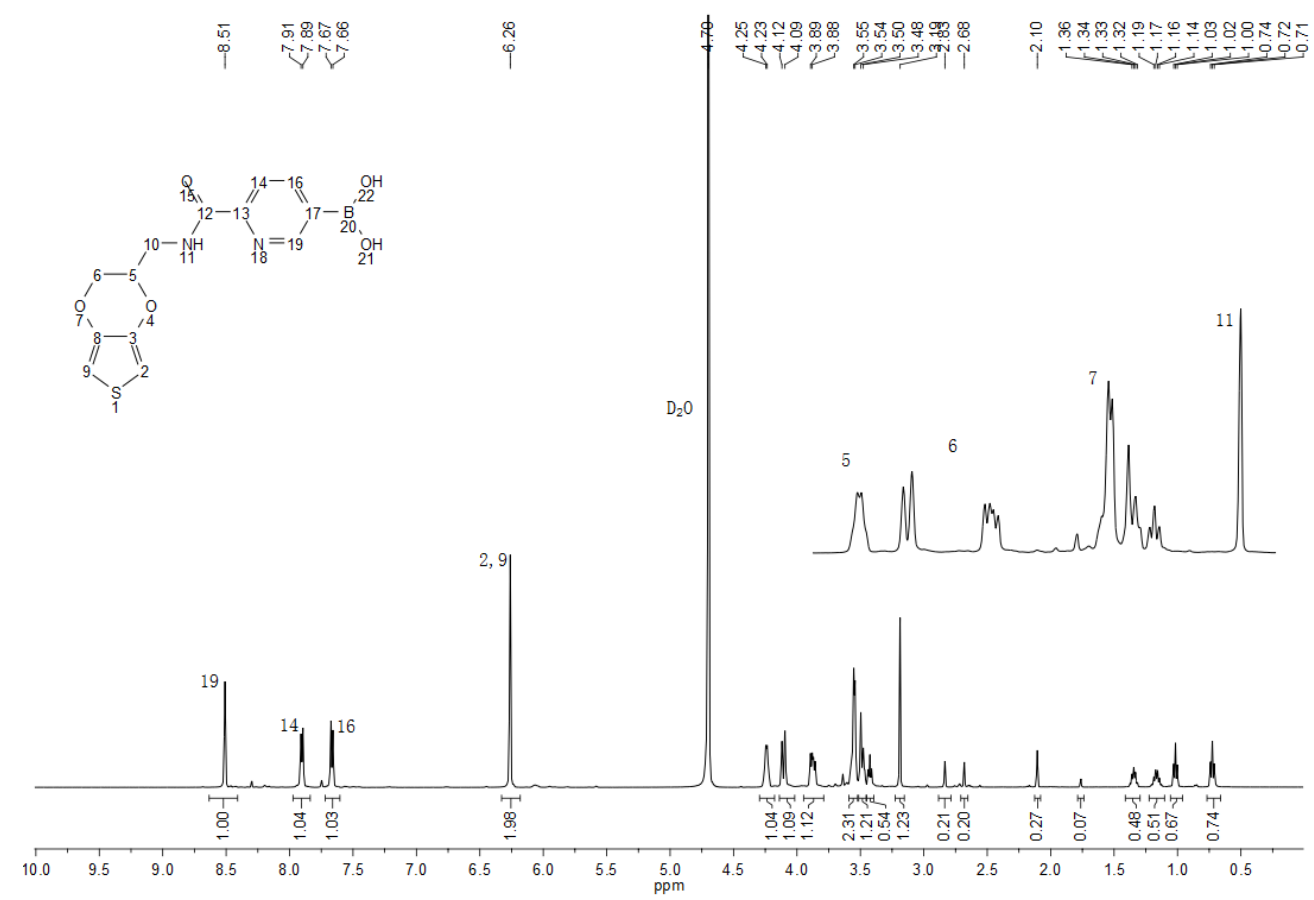

Figure S1. ${ }^{1} \mathrm{H}-\mathrm{NMR}$ spectra of EDOT-PyBA (500 MHz in $\left.\mathrm{D}_{2} \mathrm{O}\right)$. 


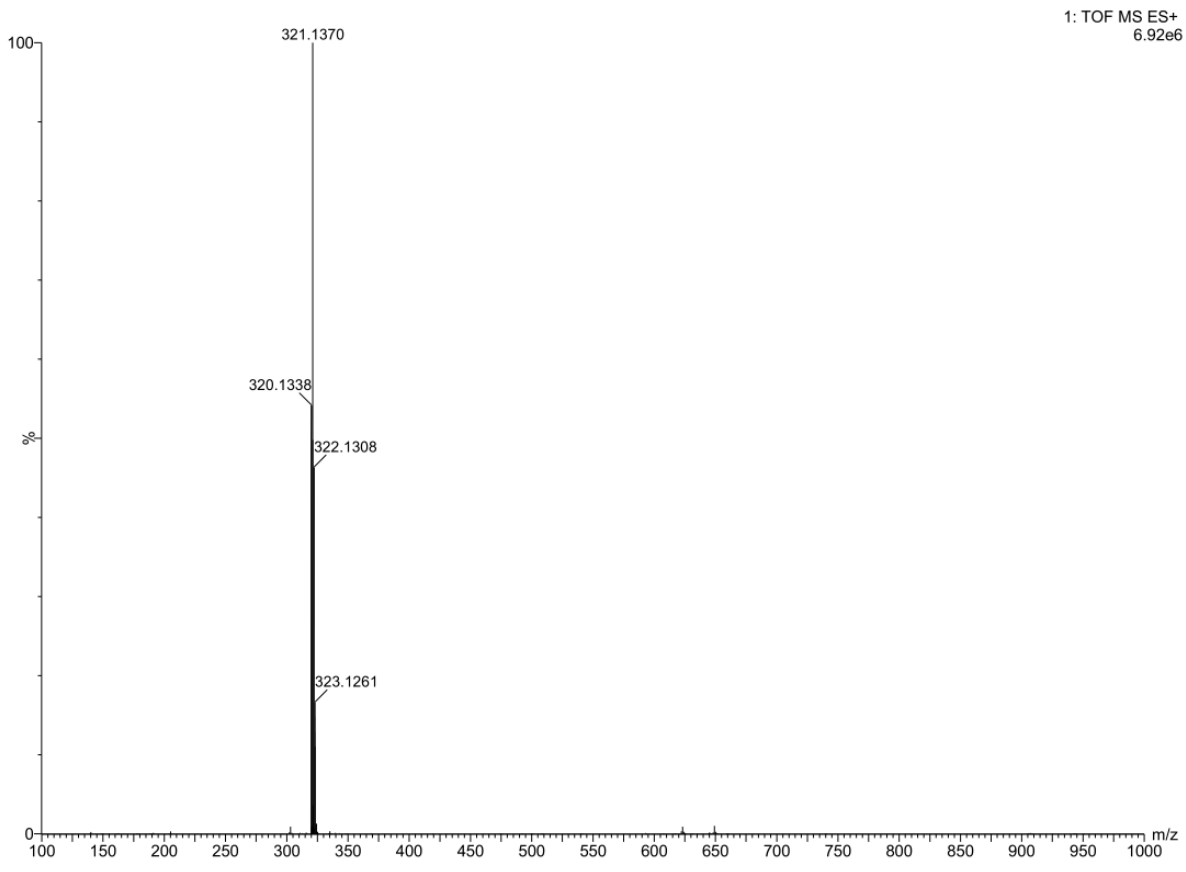

Figure S2. High resolution ESI-MS for EDOT-PyBA. Calculated $m / z$ for $\mathrm{C}_{13} \mathrm{H}_{14} \mathrm{BN}_{2} \mathrm{O}_{5} \mathrm{~S}^{+}$ $\left(\mathrm{M}+\mathrm{H}^{+}\right)$at 321.07, peak found at 321.13. 


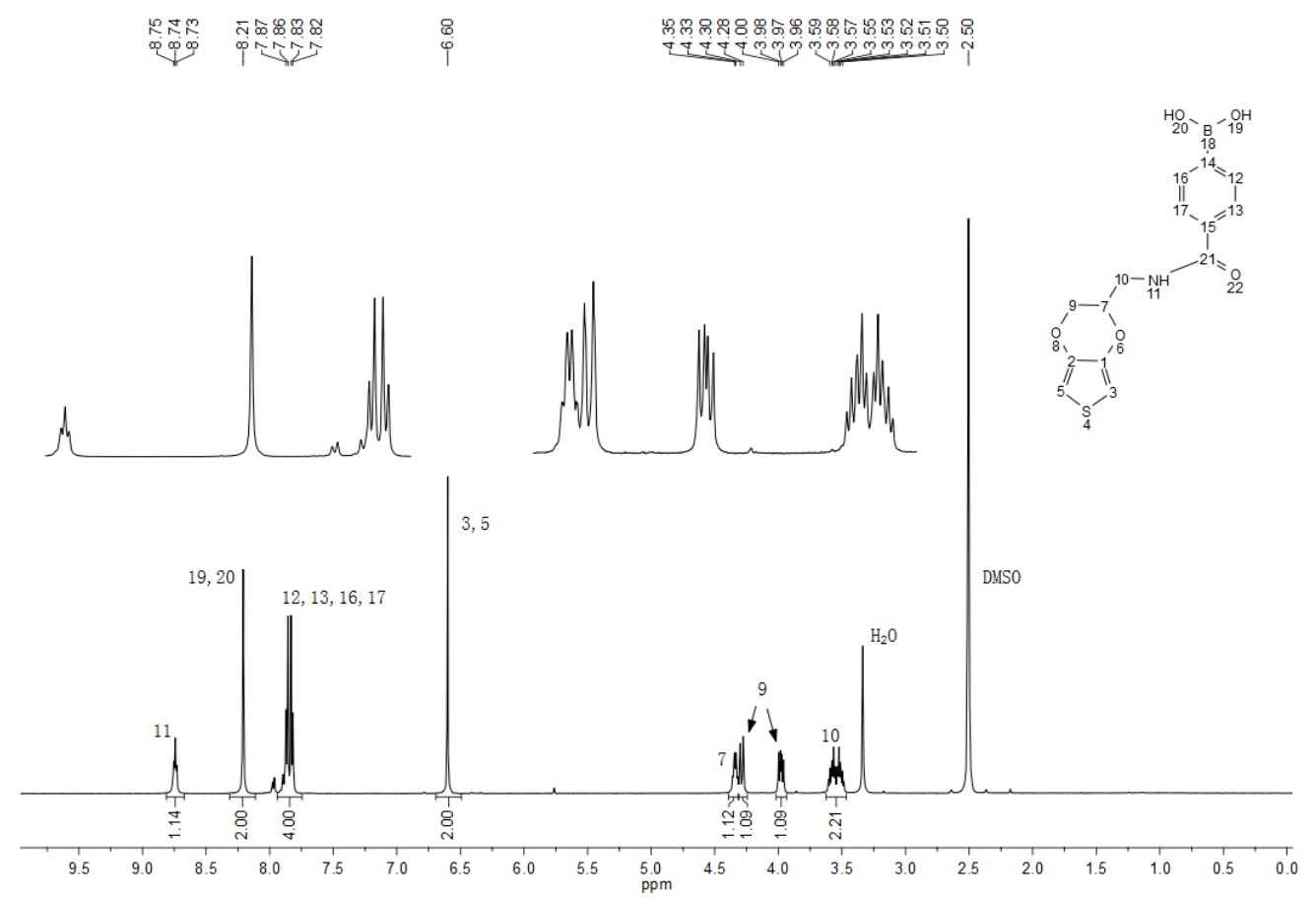

Figure S3. ${ }^{1} \mathrm{H}-\mathrm{NMR}$ spectra of EDOT-PhBA (500 MHz in DMSO). 


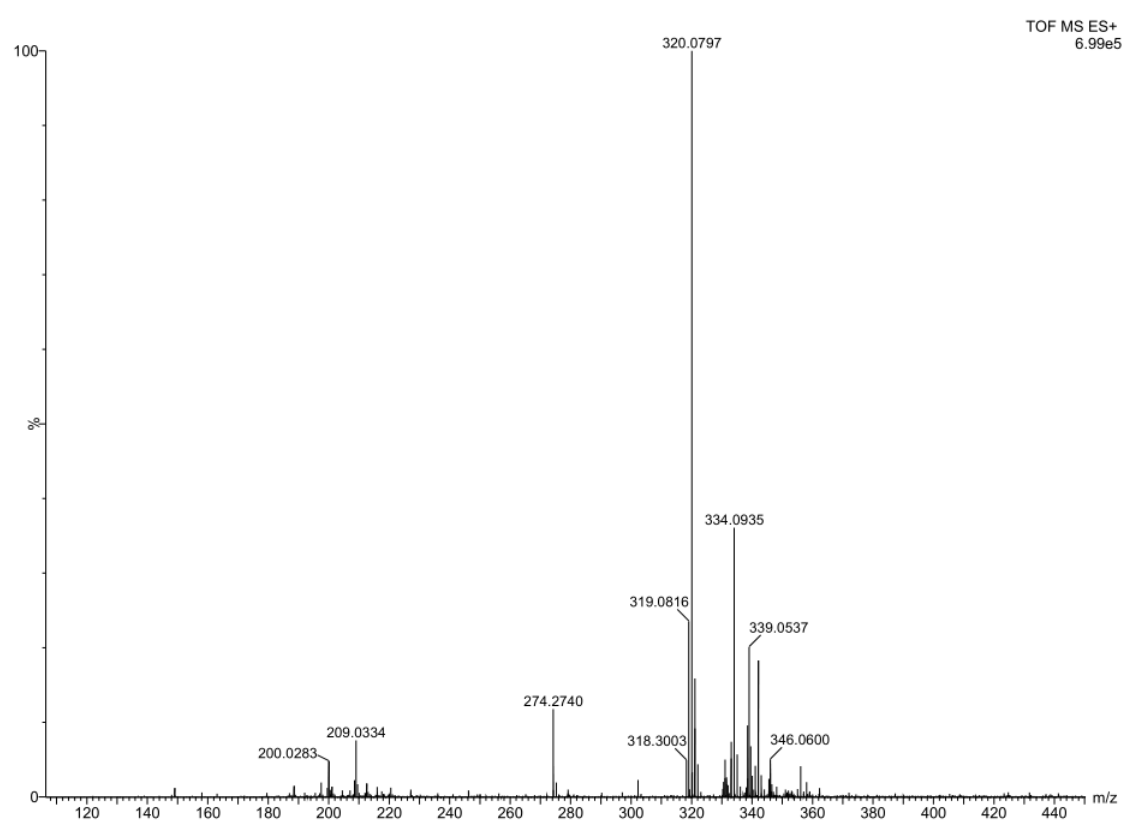

Figure S4. High resolution ESI-MS for EDOT-PhBA. Calculated $m / z$ for $\mathrm{C}_{14} \mathrm{H}_{15} \mathrm{BNO}_{5} \mathrm{~S}^{+}$ $\left(\mathrm{M}+\mathrm{H}^{+}\right)$at 320.07 , peak found at 320.07 . 
Table S1. Element proportions of poly(EDOT-PyBA) and poly(EDOT-PhBA) before and after $\mathrm{H}_{2} \mathrm{SO}_{4}$ treatment

\begin{tabular}{|l|c|c|c|c|c|c|}
\hline & \multicolumn{3}{|c|}{ poly(EDOT-PyBA) } & \multicolumn{2}{c|}{ poly(EDOT-PhBA) } \\
\hline Element proportion & $\mathrm{B} / \mathrm{C}$ & $\mathrm{N} / \mathrm{C}$ & $\mathrm{N} / \mathrm{B}$ & $\mathrm{B} / \mathrm{C}$ & $\mathrm{N} / \mathrm{C}$ & $\mathrm{N} / \mathrm{B}$ \\
\hline Before $\mathrm{H}_{2} \mathrm{SO}_{4}$ treatment & 0.06 & 0.11 & 1.83 & 0.042 & 0.039 & 0.93 \\
\hline After $\mathrm{H}_{2} \mathrm{SO}_{4}$ treatment & 0.09 & 0.14 & 1.55 & 0.059 & 0.052 & 0.88 \\
\hline
\end{tabular}



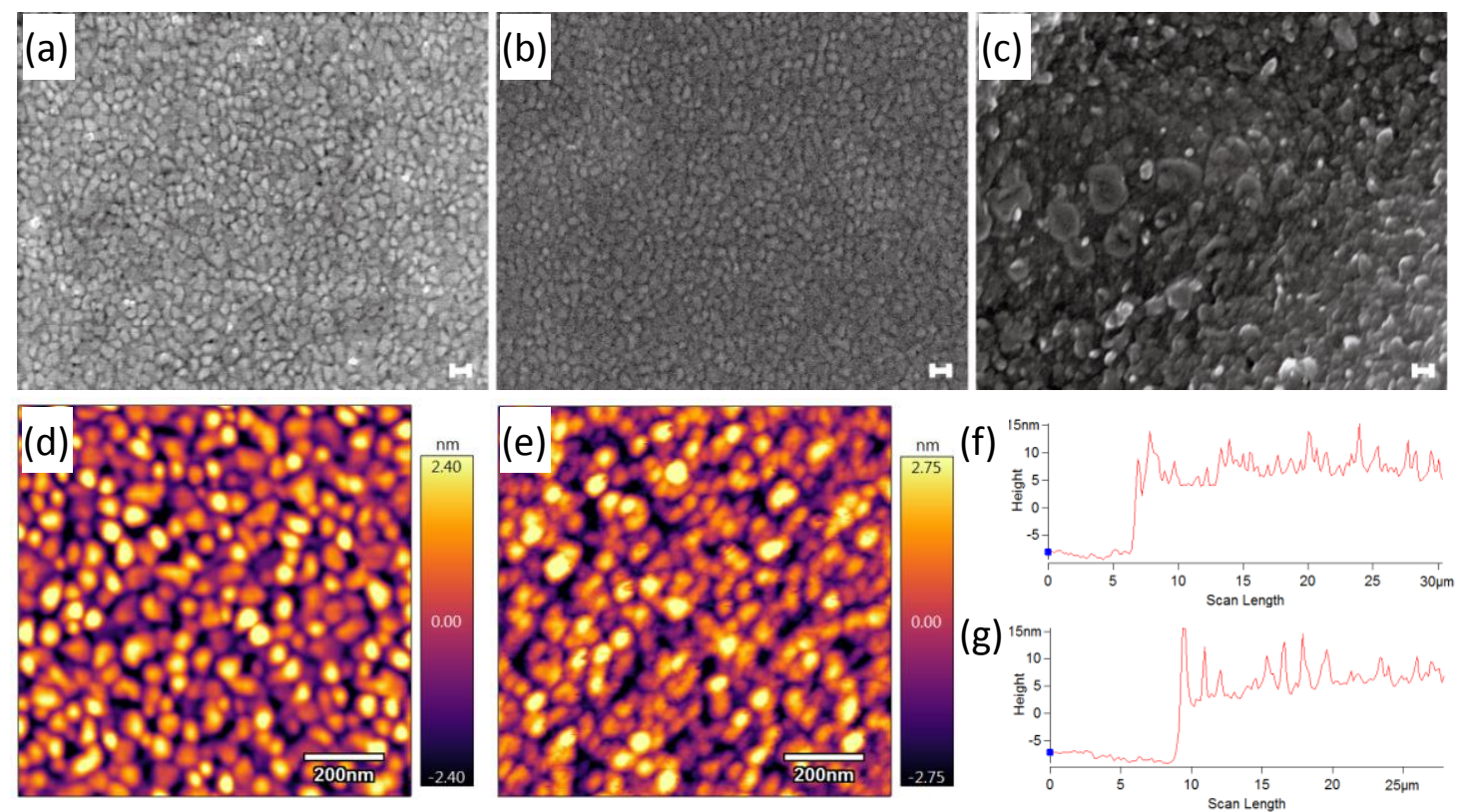

Figure S5. SEM observation for (a) Poly(EDOT-PyBA), (b) poly(EDOT-PhBA) and (c)

PEDOT films electrodeposited on planar gold electrodes. Magnification: $\times 50000$; scale bar: $100 \mathrm{~nm}$. (d) (f) AFM image $(1 \mu \mathrm{m} \times 1 \mu \mathrm{m})$ and film thickness of poly(EDOT-PyBA). (e) $(\mathrm{g})$ AFM image $(1 \mu \mathrm{m} \times 1 \mu \mathrm{m})$ and film thickness of poly(EDOT-PhBA). AFM images and film thickness were examined using Asylum Research MFP-3D Origin AFM (Oxford Instruments, UK). 

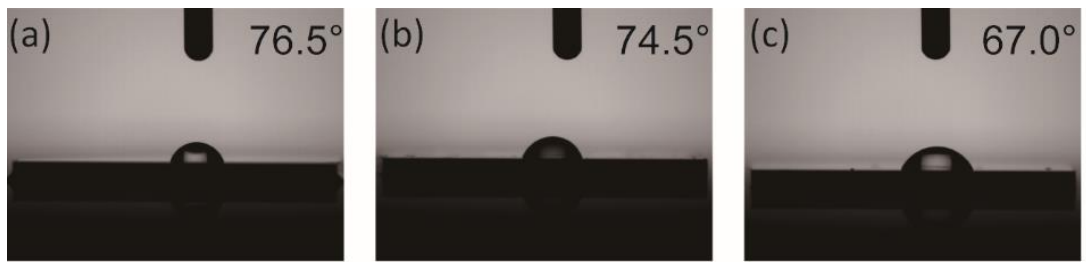

(d) $\quad 55.0^{\circ}$

Figure S6. Static water contact angles of (a) bare Au, and electrodeposited (b) PEDOT on $\mathrm{Au}$, (c) poly(EDOT-PhBA) on PEDOT/Au, and (d) poly(EDOT-PyBA) on PEDOT/Au. 

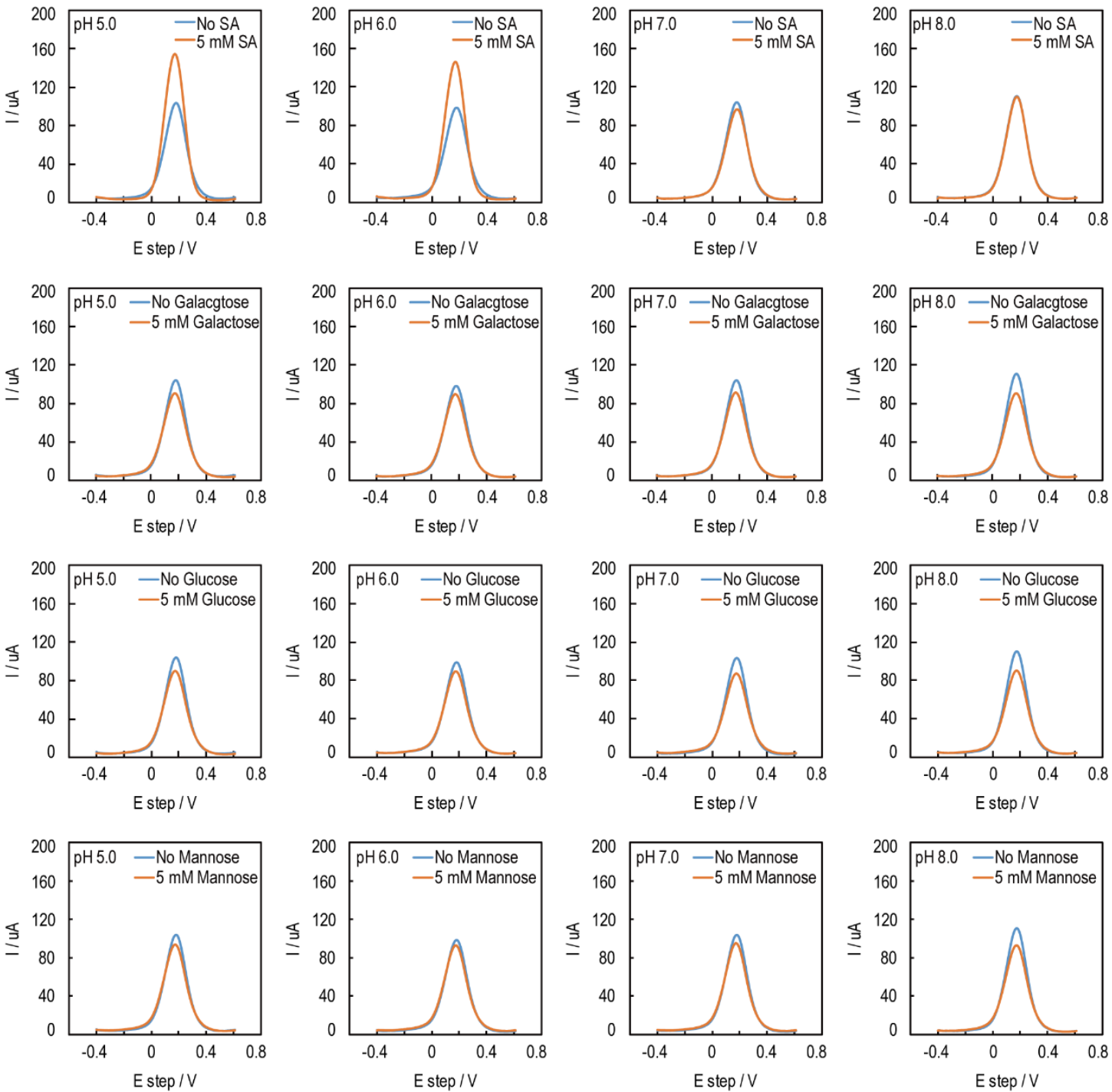

Figure S7. DPV spectra for poly(EDOT-PyBA)-deposited GC electrodes with and without $5 \mathrm{mM}$ sialic acid, glucose, galactose, or mannose in $1 \times \mathrm{DPBS}$ at $\mathrm{pH}$ 5.0-8.0. The DPV measurements were taken in the presence of $5 \mathrm{mM}$ ferri/ferro-cyanide. Counter electrode: Pt plate; Reference electrode: $\mathrm{Ag} / \mathrm{AgCl}$ wire (in $3.3 \mathrm{M} \mathrm{KCl}$ with a salt bridge). 
(a)

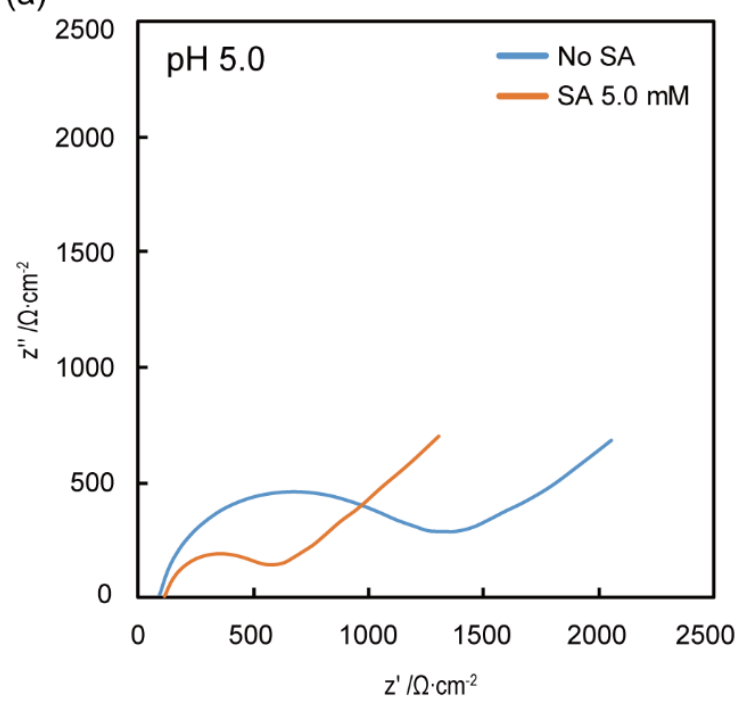

(b)

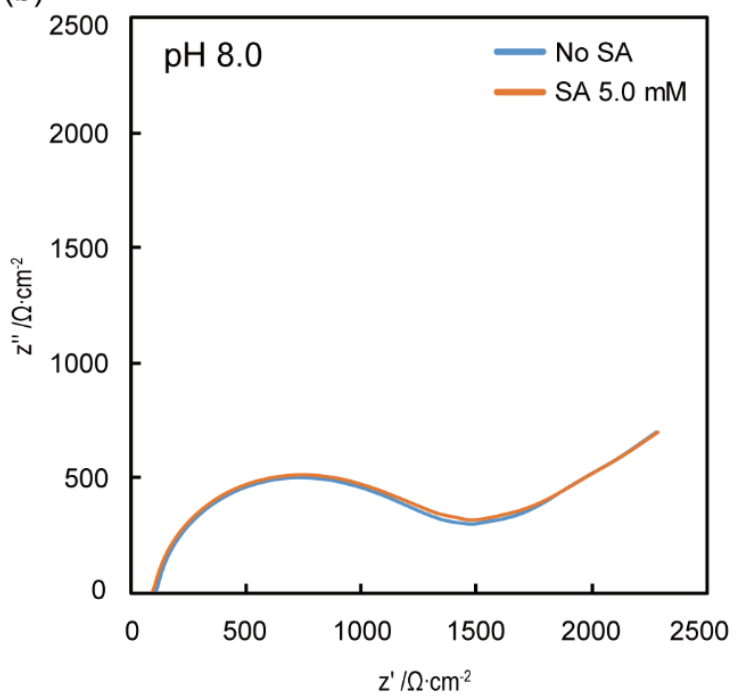

Figure S8. EIS measurement at (a) $\mathrm{pH} 5.0$ and (b) $\mathrm{pH} 8.0$ for poly(EDOT-PyBA)deposited GC electrodes in 0 and $5 \mathrm{mM}$ SA in $1 \times$ DPBS containing a redox couple of 5 $\mathrm{mM}$ ferri/ferro-cyanide. 
Table S2. Comparison of different methods for SA determination

\begin{tabular}{|c|c|c|c|c|c|}
\hline $\begin{array}{l}\text { Detection } \\
\text { method }\end{array}$ & Probe & Linear range & LOD & $\begin{array}{l}\text { Analysis } \\
\text { time }\end{array}$ & Ref \\
\hline $\begin{array}{l}\text { Fluorescent } \\
\text { sensor }\end{array}$ & $\begin{array}{l}\text { Boronic acid- } \\
\text { functionalized } \\
\text { carbon dot }\end{array}$ & $80 \mu \mathrm{M}-4 \mathrm{mM}$ & $54 \mu \mathrm{M}$ & $4 \mathrm{~min}$ & (1) \\
\hline $\begin{array}{l}\text { Fluorescent } \\
\text { sensor }\end{array}$ & $\begin{array}{l}\text { Polydiacetylene } \\
\text { liposomers }\end{array}$ & $0-0.4 \mathrm{mM}$ & $14 \mu \mathrm{M}$ & $70 \mathrm{~min}$ & (2) \\
\hline $\begin{array}{l}\text { Colorimetric } \\
\text { sensor }\end{array}$ & $\begin{array}{l}\text { Boronic acid- } \\
\text { functionalized } \\
\text { AuNPs }\end{array}$ & $80 \mu \mathrm{M}-2 \mathrm{mM}$ & $68 \mu \mathrm{M}$ & $10 \mathrm{~min}$ & (3) \\
\hline $\begin{array}{l}\text { Potentiometric } \\
\text { sensor }\end{array}$ & $\begin{array}{l}\text { poly(anilineboronic } \\
\text { acid)/graphene }\end{array}$ & $2 \mu \mathrm{M}-1.38 \mathrm{mM}$ & $0.8 \mu \mathrm{M}$ & & (4) \\
\hline $\begin{array}{l}\text { Colorimetric } \\
\text { sensor }\end{array}$ & $\begin{array}{l}\text { Boronic acid- } \\
\text { funtionalized } \\
\text { AuNPs }\end{array}$ & $0.08-0.25 \mathrm{mM}$ & $35.0 \mu \mathrm{M}$ & $10 \mathrm{~min}$ & (5) \\
\hline This work & $\begin{array}{l}\text { Poly(EDOT- } \\
\text { Pyridylboronic acid }\end{array}$ & $0.1-3.0 \mathrm{mM}$ & $0.1 \mathrm{mM}$ & $3 \min$ & \\
\hline
\end{tabular}




\section{References}

(1) Xu, S.; Che, S.; Ma, P.; Zhang, F.; Xu, L.; Liu, X.; Wang, X.; Song, D.; Sun, Y. One-step fabrication of boronic-acid-functionalized carbon dots for the detection of sialic acid. Talanta 2019, 197, 548-552.

(2) Wang, D. E.; Yan, J.; Jiang, J.; Liu, X.; Tian, C.; Xu, J.; Yuan, M. Sen; Han, X.; Wang, J. Polydiacetylene liposomes with phenylboronic acid tags: a fluorescence turn-on sensor for sialic acid detection and cell-surface glycan imaging. Nanoscale 2018, 10, 4570-4578.

(3) Sankoh, S.; Thammakhet, C.; Numnuam, A.; Limbut, W.; Kanatharana, P.; Thavarungkul, P. 4-mercaptophenylboronic acid functionalized gold nanoparticles for colorimetric sialic acid detection. Biosens. Bioelectron. 2016, 85, 743-750.

(4) Zhou, Y.; Dong, H.; Liu, L.; Liu, J.; Xu, M. A novel potentiometric sensor based on a poly(anilineboronic acid)/graphene modified electrode for probing sialic acid through boronic acid-diol recognition. Biosens. Bioelectron. 2014, 60, 231-236.

(5) Jayeoye, T. J.; Cheewasedtham, W.; Putson, C.; Rujiralai, T. A selective probe based on 3 - aminophenyl boronic acid assembly on dithiobis (succinimidylpropionate) functionalized gold nanoparticles for sialic acid detection in human serum. J. Mol. Liq. 2019, 281, 407-414. 